\title{
Evaluation of nepafenac in prevention of macular edema following cataract surgery in patients with diabetic retinopathy
}

This article was published in the following Dove Press journal:

Clinical Ophthalmology

2 August 2012

Number of times this article has been viewed

\author{
Rishi Singh' \\ Louis Alpern² \\ Glenn J Jaffe ${ }^{3}$ \\ Robert P Lehmann ${ }^{4}$ \\ John Lim $^{5}$ \\ Harvey J Reiser ${ }^{6}$ \\ Kenneth Sall ${ }^{7}$ \\ Thomas Walters ${ }^{8}$ \\ Dana Sager 9
}

'Cole Eye Institute, Cleveland Clinic Foundation, Cleveland, $\mathrm{OH}$,

${ }^{2}$ The Cataract, Glaucoma, and

Refractive Surgery Center, El Paso,

TX, ${ }^{3}$ Duke Eye Center, Duke Reading

Center, Duke University, Durham, NC,

${ }^{4}$ Lehmann Eye Center, Nacogdoches,

TX, ${ }^{5}$ Houston Eye Associates,

Houston, TX, ${ }^{6}$ Eye Care Specialists,

Kingston, PA, ${ }^{7}$ Sall Research Medical

Center, Artesia, CA, ${ }^{8}$ Texan Eye,

Austin, TX, ${ }^{9}$ Alcon Research Ltd, Fort

Worth, TX, USA
Correspondence: Rishi Singh

Cole Eye Institute, Cleveland Clinic

Foundation, 9500 Euclid Avenue, i-32

Cleveland, OH 44195, USA

Email drrishisingh@gmail.com
Background: The purpose of this study was to evaluate nepafenac ophthalmic suspension $0.1 \%$ (Nevanac ${ }^{\circledR}$; Alcon Research Ltd) in the prevention of macular edema following cataract surgery in diabetic retinopathy patients.

Methods: This was a multicenter, randomized, double-masked, vehicle-controlled study of 263 adult diabetic patients with nonproliferative diabetic retinopathy requiring cataract surgery. Patients were randomized (1:1) to instill nepafenac or vehicle three times daily beginning 1 day prior to surgery through day 90 . Efficacy included the percentage of patients who developed macular edema ( $\geq 30 \%$ increase in central subfield macular thickness from baseline) and the percentage of patients with decreases of more than five letters in best-corrected visual acuity from day 7 to 90 .

Results: A significantly lower percentage of patients in the nepafenac group developed macular edema relative to patients in the vehicle group (3.2\% versus $16.7 \% ; P<0.001)$. A significantly lower percentage of patients in the nepafenac group had best-corrected visual acuity decreases of more than five letters relative to patients in the vehicle group on day $30(P<0.001)$, day 60 $(P=0.002)$, and day $90(P=0.006)$. The mean central subfield macular thickness and mean percent change from baseline in macular volume were also significantly lower in the nepafenac group versus the vehicle group at days 14 through $90(P \leq 0.005)$. No safety issues or trends were identified when dosing was increased to 90 days that negatively impacted the favorable benefit/risk profile of nepafenac.

Conclusion: Nepafenac demonstrated statistically significant and clinically relevant advantages compared with vehicle in preventing macular edema and maintaining visual acuity in diabetic patients following cataract surgery. These advantages were seen at multiple time points over the course of the 90-day therapy period. There was no clinically relevant increase in risk from 90 days dosing compared with 14 days. Therefore, with a similar safety profile and benefit in preventing macular edema and maintaining vision, the risk/benefit to the diabetic patient undergoing cataract surgery appears to be positive.

Keywords: cataract extraction, diabetes, macular edema, nonsteroidal anti-inflammatory drug, topical, ocular surgery, retinopathy

\section{Introduction}

Macular edema is a common cause of poor visual outcome following uneventful cataract surgery ${ }^{1}$ that has been reported to occur in up to $2 \%$ of patients. ${ }^{2-4}$ The incidence may be as high as $20 \%$ when cataract extraction is complicated by posterior capsule rupture with vitreous loss or severe iris trauma. ${ }^{1}$ Further, it is well documented that macular changes are more likely to occur following cataract surgery in diabetic 
patients, especially those with pre-existing retinopathies, compared with nondiabetic patients. ${ }^{5-8}$ Estimates of the rate of development of macular edema in diabetic populations (with or without diabetic retinopathy) vary across studies, ranging from $31 \%$ to $81 \%$ at various time points following

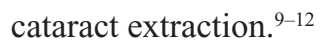

Factors involved in the pathogenesis of diabetic macular edema include chronic hyperglycemia, blood-retinal barrier dysfunction, and chronic subclinical inflammation. ${ }^{8}$ Postsurgical inflammation, in particular, is believed to be a major factor in macular edema, that develops subsequent to cataract extraction. Prostaglandins contribute substantially to the inflammatory processes that result in fluid leakage from perifoveal capillaries into the extracellular space of the macular region. ${ }^{13}$ Given that topical nonsteroidal anti-inflammatory drugs (NSAIDs) block the cyclooxygenase enzymes responsible for prostaglandin production, some reports suggest NSAIDs may also reduce the incidence, duration, and severity of macular edema. ${ }^{13-17}$

Nepafenac ophthalmic suspension $0.1 \%$ (Nevanac ${ }^{\circledR}$; Alcon Research Ltd, Fort Worth, TX) is a topical NSAID indicated in the United States for the treatment of pain and inflammation associated with cataract surgery. Nepafenac is a prodrug that rapidly penetrates the cornea and is deaminated to form the active metabolite, amfenac, by intraocular hydrolases within ocular tissues, including the ciliary body epithelium, retina, and choroid. ${ }^{18}$ Because bioconversion is targeted to the iris/ciliary body, and to a greater extent to the retina and choroid, nepafenac may have prolonged activity in the vascularized tissues of the eye. ${ }^{19}$ Nepafenac and amfenac are potent inhibitors of the cyclooxygenase enzyme isoforms (COX1 and COX2). ${ }^{20}$ In a rabbit model of concanavalin A-induced retinal inflammation, topical nepafenac penetrated into the posterior segment, where it decreased vitreous protein and prostaglandin $\mathrm{E}_{2}$ concentrations and inhibited the breakdown of the blood-retina barrier. ${ }^{21}$ In the same study, other NSAIDs, including ketorolac and diclofenac, failed to inhibit accumulation of these inflammatory markers.

The objective of this study was to determine whether treatment with nepafenac for up to 90 days after cataract surgery prevents macular edema and the associated loss of visual acuity in diabetic patients. In particular, the study evaluated the percentages of patients who developed macular edema (defined as $\geq 30 \%$ increase in central subfield macular thickness relative to the presurgical baseline measurement) 30,60, and 90 days following cataract surgery, and evaluated postsurgical visual acuity changes at those same time points.

\section{Materials and methods Study design}

This was a multicenter, randomized, double-masked, vehiclecontrolled, parallel-group study designed to evaluate the safety and efficacy of nepafenac when used for 90 days following cataract surgery in patients with diabetic retinopathy. The study consisted of nine visits, which included the screening and baseline visits (both conducted within 2 days to 4 weeks prior to the surgery visit), the cataract surgery visit (day 0), and 6 postsurgical follow-up visits (on days 1, 7, 14, 30, 60, and 90 [or early exit]).

The study was conducted in accordance with good clinical practices and the ethical principles described within the Declaration of Helsinki. All participating patients provided their written informed consent. The study protocol and associated informed consent forms were reviewed and approved for each investigational center by an institutional review board. This study is registered at clinicaltrials.gov as NCT00782717.

Enrolled patients were randomized (1:1) to receive nepafenac or vehicle and started instilling the study drug into the study eye three times daily on the day prior to cataract surgery (day -1 ); dosing continued on the day of surgery (day 0 ) and for 90 days thereafter (note that nepafenac is approved in the United States for dosing through 2 weeks [14 days] postoperatively in the treatment of pain and inflammation associated with cataract surgery). Other than the extended duration of treatment evaluated in this study, the dosing regimen described herein is consistent with administration of nepafenac for the approved indication. On the day of surgery, designated study personnel instilled one drop of the assigned study drug into each patient's study eye 30-120 minutes prior to surgery; the patients self-administered the study drug at all other times. In addition to using the assigned study drug, patients also instilled prednisolone acetate ophthalmic suspension (Omnipred ${ }^{\mathrm{TM}}$; Alcon Research Ltd) into the study eye four times daily for 2 weeks postsurgery or longer if considered necessary to treat anterior segment inflammation.

Patients were evaluated for safety and efficacy on days 1 (the day following surgery), 7, 14, 30, 60, and 90 (or at the time of early discontinuation from the study). The investigators were allowed to discontinue patients from the study and begin rescue therapy if patients developed a 30\% increase in central subfield macular thickness, developed cysts, or experienced a decrease in visual acuity associated with macular thickening. 


\section{Patients}

The study population included male and female diabetic (type 1 or type 2) patients of any race/ethnicity who were aged 18 years and older. The patients had to have had an existing diagnosis of nonproliferative diabetic retinopathy that required cataract extraction with planned implantation of a posterior chamber intraocular lens. At least $50 \%$ of all enrolled patients were required to have moderate to severe nonproliferative diabetic retinopathy, as defined by the International Clinical Diabetic Retinopathy Disease Severity Scale. ${ }^{22}$ Because this was the first study involving long-term use of nepafenac in diabetic patients, the potential for corneal problems was considered a safety concern. Therefore, eligible patients could not have had significant corneal staining scores at baseline and must not have had histories of dry eye syndrome. Patients were also excluded for other conditions that may have caused macular edema, including preexisting histories of retinal vein occlusions, ocular surgeries, inflammatory eye diseases, ocular infections, congenital ocular anomalies, and ocular traumas. Further, to ensure that patients entering the study did not have pre-existing macular edema, a reading center (Duke Reading Center, Durham, NC) performed an expedited review of optical coherence tomography (OCT) images collected at screening from each patient to confirm, prior to randomization, that the patient had a central subfield macular thickness less than 250 microns. The purpose of this review was also to identify and exclude patients who had baseline cysts, and the presence of macular traction and epiretinal membranes, which would confound retinal thickness measurements. Finally, patients were prohibited from using concomitant medications such as topical or systemic NSAIDs and steroids, which might have interfered with the assessment of the study outcome measures.

\section{Efficacy}

Macular thickness and volume were measured using optical coherence tomography (OCT; Zeiss Stratus OCT 3; Carl Zeiss Meditec, Dublin, CA). A fast macular thickness map to determine macular thickness and volume and a macular thickness map to assess macular morphology were obtained with software version 4.0, 5.0, or 6.0. Macular thickness and volume were determined for both eyes at screening, as well as in the study eye on days $7,14,30,60$, and 90 (or early exit). The central subfield measurement was calculated from approximately 128 thickness points within the 1000 micron subfield centered over the fovea or central macula. Thickness results were reported for the foveal center point, the foveal central $1 \mathrm{~mm}$ subfield, and the inner and outer sectors, each of which were divided into four quadrants (temporal, superior, nasal, and inferior). Total macular volume was determined from a $6 \mathrm{~mm}$ diameter circle centered on the foveal center. Morphological features, including intraretinal cysts, were analyzed by the reading center in a masked fashion.

Best-corrected visual acuity was assessed by a certified technician and refractionist using a standardized Early Treatment Diabetic Retinopathy Study chart at $4 \mathrm{~m}$ or $1 \mathrm{~m}$ (as necessary) in both eyes at baseline and day 90 (or early exit), as well as in the study eye on days 1, 7, 14, 30, and 60 .

\section{Safety}

Information on adverse events was collected for all patients after the first administration of study drug on day -1 and continuing through day 90 (or early exit). All adverse events were assessed according to their seriousness, severity (mild, moderate, or severe), relationship to the study drug, individual characteristics (eg, onset, duration, and outcome), and whether or not they resulted in patient discontinuation from the study. It should be noted that surgery-related postoperative conditions that are normal consequences of ocular surgery and not considered clinically relevant were only reported as adverse events at the discretion of the investigator. Further, study personnel at each investigational center who were responsible for administering the study medication on day 0 did not assess adverse events or the corneal staining results. Patients who experienced corneal erosion (defined as a loss of corneal tissue with stromal thinning, with or without infiltrates [excluding dellen formation]) at any time following instillation of the study drug were to have immediately discontinued use of the drug and were to have been discontinued from the study.

In addition to a review of reported adverse events, the safety of nepafenac relative to the vehicle was assessed by direct routine ocular examination. The safety review included an evaluation of reported ocular signs (inflammatory cells and flare, corneal edema, and bulbar conjunctival injection), intraocular pressure measurements, dilated fundus parameters (retina/macula/choroid and optic nerve), and corneal fluorescein staining scores.

\section{Statistical analysis}

All statistical analyses were performed using SAS software (SAS Institute, Cary, NC). Primary study inferences were based on testing of superiority conducted with the intent-to-treat analysis set, which included all patients who were exposed to the study drug, completed the implant surgery, and had at least one on-therapy postsurgical visit at which OCT was performed. Safety was evaluated for all 
patients who received exposure to the study drug or potential exposure to the study drug (ie, patients who discontinued the study prior to surgery, but returned an opened bottle of study drug). The last observation carried forward method was used to impute missing macular thickness and best-corrected visual acuity data; no imputations for missing data were made in the safety analyses.

\section{Primary outcome measure}

The primary objective was to demonstrate the superiority of nepafenac relative to vehicle based on the percentage of diabetic retinopathy patients who developed macular edema within 90 days following cataract surgery; in this study, macular edema was defined as an increase of $30 \%$ or more in central subfield macular thickness relative to the presurgical baseline measurement. For the primary efficacy evaluation, the treatment groups were compared with statistical inferences drawn at a two-sided alpha level of 0.05 .

\section{Secondary outcome measure}

The secondary efficacy variable was the percentage of patients who had decreases of more than five letters in bestcorrected visual acuity from day 7 (postsurgical baseline) to day 90 (or early exit). For the secondary endpoint, the treatment groups were compared with statistical inferences drawn if the associated $P$ value was less than 0.05 and the primary efficacy endpoint was also significant at the same level.

\section{Exploratory outcome measures}

Planned exploratory analyses were conducted to evaluate the mean changes in best-corrected visual acuity from the postsurgical baseline to day 90. Post hoc exploratory analyses were conducted to evaluate percentages of patients with diabetic retinopathy who developed macular edema within 30 days and 60 days following cataract surgery, mean central subfield macular thickness measurement, mean changes from baseline in central subfield macular thickness measurements, and percent changes from baseline in macular volume measurements. Additionally, post hoc evaluations included mean best-corrected visual acuity result by visit, categorical changes (including increases and decreases of 15 letters read or more) in best-corrected visual acuity from the postsurgical baseline to day 90, percentage of patients who had decreases of more than five letters in best-corrected visual acuity from the postsurgical baseline to both day 30 and day 60, and percentage of nepafenac-treated patients who returned to within $10 \%$ of their baseline central subfield value by day 60 and by day 90 .

\section{Results \\ Patient characteristics and disposition}

Between November 2008 and July 2010, 263 patients were enrolled into this study at 41 investigational centers in the United States. Of the enrolled patients, 133 received nepafenac and 130 received vehicle; 251 patients were included in the intent-to-treat analysis set and 253 patients were included in the safety analysis set.

Patients in the intent-to-treat analysis set had a mean age of $66.5 \pm 9.4$ years, and were predominantly female $(62.9 \%)$ and white $(81.7 \%)$; the patients primarily had brown eyes $(69.7 \%)$ and moderate to severe nonproliferative diabetic retinopathy $(74.1 \%)$. Prior to surgery (ie, the presurgical baseline), the enrolled patients had a mean central subfield macular thickness of $200.8 \pm 25.8 \mu \mathrm{m}$ and a mean best-corrected visual acuity of $67.4 \pm 12.0$ letters read. On day 7 (ie, the postsurgical baseline), the patients had a mean best-corrected visual acuity of $80.9 \pm 7.4$ letters read. The demographic and baseline characteristics of the patients in the nepafenac and vehicle groups were generally similar (Table 1).

\section{Macular edema, macular thickness, and macular volume}

A significantly greater percentage of patients in the vehicle group developed macular edema compared with the percentage of patients in the nepafenac group when evaluated following cataract surgery at day $30(8.7 \%$ versus $2.4 \%$, respectively; $P=0.029)$, day 60 (15.1\% versus $2.4 \%$, respectively; $P<0.001$ ), and day 90 (16.7\% versus $3.2 \%$, respectively; $P<0.001$, Table 2 ). The result on day 90 was the primary outcome measure.

At the postsurgical study visits conducted on days 14, 30, 60, and 90, mean central subfield macular thickness measurements were significantly lower in patients who received nepafenac than in patients who received vehicle $(P<0.001$ for each pairwise comparison, Figure 1$)$. The mean maximum change from baseline to any study visit in macular thickness was significantly lower for patients in the nepafenac group compared with patients in the vehicle group (mean for each respective group, $18.9 \pm 19.5 \mu \mathrm{m}$ and $40.8 \pm 49.0 \mu \mathrm{m} ; P<0.001)$. Finally, on postsurgical days 14 , 30,60 , and 90 , the mean percent changes in macular volume from baseline to each study visit were significantly lower in the nepafenac group than in the vehicle group (day 14, $P=0.005$; days 30,60, and 90, $P<0.001$, Figure 2). Thus, this study demonstrates that the incidence of macular edema was reduced and changes in macular thickening and volume 
Table I Demographic and baseline characteristics by treatment group

\begin{tabular}{|c|c|c|c|}
\hline & $\begin{array}{l}\text { Total } \\
(n=25 I)\end{array}$ & $\begin{array}{l}\text { Nepafenac } \\
(n=125)\end{array}$ & $\begin{array}{l}\text { Vehicle } \\
(n=126)\end{array}$ \\
\hline \multicolumn{4}{|l|}{ Sex (n, \%) } \\
\hline Male & $93(37.1)$ & $42(33.6)$ & $51(40.5)$ \\
\hline Female & $158(62.9)$ & $83(66.4)$ & $75(59.5)$ \\
\hline \multicolumn{4}{|l|}{ Age (years) } \\
\hline Mean (SD) & $66.5(9.4)$ & $66.6(9.3)$ & $66.4(9.7)$ \\
\hline (minimum, maximum) & $(32,87)$ & $(39,87)$ & $(32,84)$ \\
\hline \multicolumn{4}{|l|}{ Ethnicity $(n, \%)$} \\
\hline $\begin{array}{l}\text { Hispanic, Latino, } \\
\text { or Spanish }\end{array}$ & $87(34.7)$ & $42(33.6)$ & $45(35.7)$ \\
\hline $\begin{array}{l}\text { Not Hispanic, Latino, } \\
\text { or Spanish }\end{array}$ & $164(65.3)$ & $83(66.4)$ & $81(64.3)$ \\
\hline \multicolumn{4}{|l|}{ Race (n, \%) } \\
\hline \multicolumn{4}{|l|}{ or Alaska Native } \\
\hline Asian & $8(3.2)$ & $7(5.6)$ & $\mathrm{I}(0.8)$ \\
\hline Black or African & $34(13.5)$ & $21(16.8)$ & $13(10.3)$ \\
\hline \multicolumn{4}{|l|}{ American } \\
\hline Other & $3(1.2)$ & $0(0.0)$ & $3(2.4)$ \\
\hline White & $205(81.7)$ & $97(77.6)$ & $108(85.7)$ \\
\hline \multicolumn{4}{|l|}{ Iris color (n, \%) } \\
\hline Blue & $42(16.7)$ & $20(16.0)$ & $22(17.5)$ \\
\hline Brown & $175(69.7)$ & $88(70.4)$ & $87(69.0)$ \\
\hline Green & $7(2.8)$ & $2(1.6)$ & $5(4.0)$ \\
\hline Grey & $3(1.2)$ & $2(1.6)$ & $\mathrm{I}(0.8)$ \\
\hline Hazel & $24(9.6)$ & $13(10.4)$ & II (8.7) \\
\hline \multicolumn{4}{|c|}{ NPDR classification (n, \%) } \\
\hline Mild NPDR & 65 (25.9) & $33(26.4)$ & $32(25.4)$ \\
\hline Moderate NPDR & $165(65.7)$ & $82(65.6)$ & $83(65.9)$ \\
\hline Severe NPDR & $21(8.4)$ & $10(8.0)$ & II (8.7) \\
\hline \multicolumn{4}{|c|}{ Central subfield macular thickness (microns) } \\
\hline Mean (SD) & $200.8(25.8)$ & $197.9(26.6)$ & $203.6(24.8)$ \\
\hline (minimum, maximum) & $(127,280)$ & $(127,280)$ & $(134,248)$ \\
\hline \multicolumn{4}{|c|}{ BCVA (letters read): presurgical baseline } \\
\hline $\mathrm{N}$ & 249 & 125 & 124 \\
\hline Mean (SD) & $67.4(12.0)$ & $68.2(9.3)$ & $66.7(14.1)$ \\
\hline (minimum, maximum) & $(15,99)$ & $(37,88)$ & $(15,99)$ \\
\hline \multicolumn{4}{|c|}{ BCVA (letters read): postsurgical baseline (day 7) } \\
\hline $\mathrm{N}$ & 247 & 124 & 123 \\
\hline Mean (SD) & $80.9(7.4)$ & $81.3(7.5)$ & $80.5(7.3)$ \\
\hline (minimum, maximum) & $(41,100)$ & $(41,99)$ & $(56,100)$ \\
\hline
\end{tabular}

Note: Baseline data was obtained at the presurgical baseline visit unless otherwise stated.

Abbreviations: BCVA, best corrected visual acuity; NPDR, nonproliferative diabetic retinopathy; PDR, proliferative diabetic retinopathy; SD, standard deviation.

were less severe among patients in the nepafenac group when compared with patients in the vehicle group.

\section{Best-corrected visual acuity}

The percentages of patients who experienced visual acuity losses of more than five letters from the postsurgical baseline to days 30,60, and 90 were consistently smaller in the nepafenac group than in the vehicle group (day 30, 2.4\%
Table 2 Development of macular edema after cataract surgery

\begin{tabular}{|c|c|c|c|c|c|}
\hline \multirow[t]{2}{*}{ Macular edema ${ }^{a}$} & \multicolumn{2}{|c|}{$\begin{array}{l}\text { Nepafenac } \\
n=125\end{array}$} & \multicolumn{2}{|c|}{$\begin{array}{l}\text { Vehicle } \\
n=I 26\end{array}$} & \multirow[t]{2}{*}{$P$-value } \\
\hline & $n$ & $(\%)$ & $n$ & (\%) & \\
\hline Within 30 days & 3 & (2.4) & II & $(8.7)$ & 0.029 \\
\hline Within 60 days & 3 & $(2.4)$ & 19 & $(15.1)$ & $<0.001$ \\
\hline Within 90 days & 4 & (3.2) & 21 & $(16.7)$ & $<0.00 \mathrm{I}$ \\
\hline
\end{tabular}

Notes: aMacular edema was defined as $\geq 30 \%$ increase from the presurgical baseline in central subfield macular thickness as measured by optical coherence tomography (Stratus OCT 3); ' $P$-value was calculated from a chi-square test.

versus $14.8 \%$, respectively; $P<0.001$; day $60,2.4 \%$ versus $13.1 \% ; P=0.002$; and day $90,5.6 \%$ versus $11.5 \% ; P=0.102$, Table 3 ). The result on day 90 was the secondary outcome measure.

At day 90, seven patients $(5.6 \%)$ in the nepafenac group experienced visual acuity losses from the postsurgical baseline of more than five letters. However, the visual acuity loss in four of these patients was clearly unrelated to macular edema; two patients developed posterior capsular opacification, one patient had clinically significant superficial punctate keratitis, and one patient had a vitreous hemorrhage that resulted in a 74-letter decrease in best-corrected visual acuity. There were no similarly confounding reasons for bestcorrected visual acuity losses among patients in the vehicle group. When the result at day 90 was corrected to discount the aforementioned patients in the nepafenac group, the difference in the percentages of patients in each group who experienced visual acuity losses of more than five letters was significant $(2.5 \%$ versus $11.5 \% ; P=0.006$, Table 3 ).

Overall, beginning with postsurgical day 14 and continuing through the end of the study, the mean number of letters read at each study visit was greater for patients in the nepafenac group than for patients in the vehicle group (Figure 3). While the mean change in best-corrected visual acuity from the postsurgical baseline to day 90 was similar in each treatment group $(P=0.226)$, a significantly greater percentage of patients in the nepafenac group than in the vehicle group experienced improvements of 15 letters or more from the postsurgical baseline to day 90 (56.8\% versus $41.9 \%$, respectively; $P=0.019$, Table 4 ). Thus, patients in the nepafenac group generally maintained better best-corrected visual acuity after cataract surgery when compared with patients in the vehicle group.

\section{Subgroup and additional evaluations}

In general, while the number of patients who developed macular edema during the study was small, no trends indicating greater or lesser efficacy were observed in the 


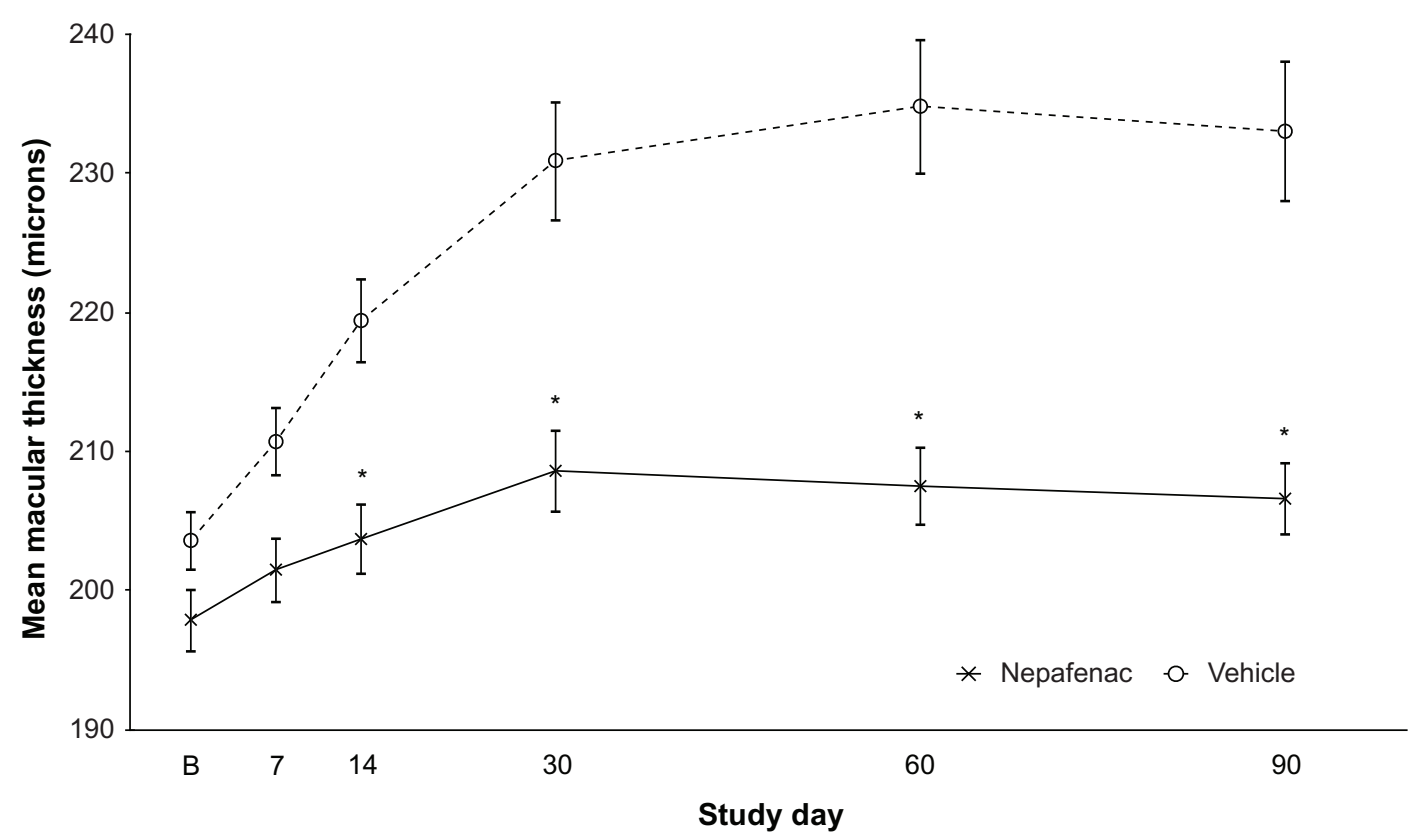

Figure I Mean central subfield macular thickness (intent-to-treat data).

Notes: Data are presented as the mean thickness in microns \pm standard error. The study day identified as "B" represents the presurgical baseline. The sample sizes in the nepafenac group were 125 (baseline, and days 14,30,60, and 90) and I2I (day 7); the sample sizes in the vehicle group were I26 (baseline, and days 30, 60, and 90), I 24 (day 7), and I 25 (day 14). *On days I4, 30,60, and 90, the difference between nepafenac and vehicle is significant $(P<0.00 \mathrm{I}$ in all comparisons based on a repeatedmeasures analysis of variance).

evaluation of the study drugs by patient age, sex, ethnicity, or nonproliferative diabetic retinopathy classification (Table 5). While the majority of patients in each treatment group had a nonproliferative diabetic retinopathy severity of moderate at baseline, the percentage of patients who developed macular edema within 90 days postsurgery increased with nonproliferative diabetic retinopathy severity regardless of treatment group. However, a greater increase was observed in the vehicle group relative to the nepafenac group.

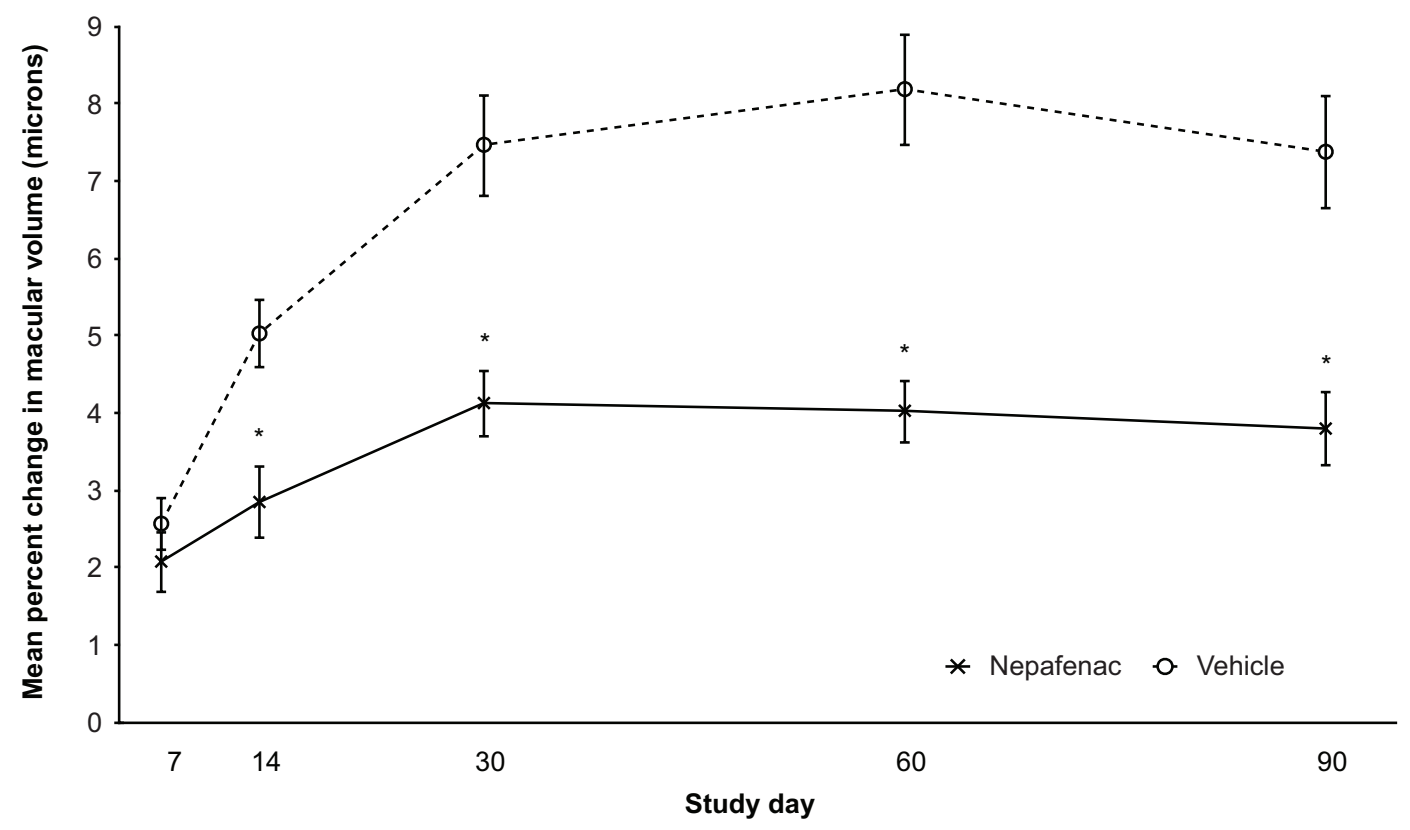

Figure 2 Mean percent change from presurgical baseline in macular volume (intent-to-treat data)

Notes: Data are presented as the mean percent change in volume \pm standard error. The sample sizes in the nepafenac group were 121 (day 7) and 125 (days 14, 30, 60, and 90); the sample sizes in the vehicle group were I 24 (day 7), I25 (day 14), and I26 (days 30, 60, and 90). *On days I4, 30, 60, and 90 , the difference between nepafenac and vehicle is significant $(P=0.005$ for day 14 and $P<0.001$ for days 30,60 , and 90 , based on a repeated-measures analysis of variance). 
Table 3 Best-corrected visual acuity decreases of more than 5 letters

\begin{tabular}{|c|c|c|c|c|c|}
\hline \multirow{2}{*}{$\begin{array}{l}\text { Visual acuity } \\
>5 \text { letters decrease } \\
\text { from day } 7\end{array}$} & \multicolumn{2}{|c|}{$\begin{array}{l}\text { Nepafenac } \\
n=124\end{array}$} & \multicolumn{2}{|c|}{$\begin{array}{l}\text { Vehicle } \\
n=122\end{array}$} & \multirow[t]{2}{*}{$P$-value ${ }^{a}$} \\
\hline & $\bar{n}$ & (\%) & $\bar{n}$ & (\%) & \\
\hline Day 30 visit & 3 & $(2.4)$ & 18 & $(14.8)$ & $<0.001$ \\
\hline Day 60 visit & 3 & $(2.4)$ & 16 & $(13.1)$ & 0.002 \\
\hline Day 90 visit & 7 & (5.6) & 14 & $(\mathrm{I} 1.5)$ & 0.102 \\
\hline Day 90 visit - corrected ${ }^{b}$ & 3 & $(2.5)$ & 14 & $($ (II.5) & 0.006 \\
\hline
\end{tabular}

Notes: ap-value was calculated from a chi-square test; 'The corrected day 90 data exclude 4 patients in the nepafenac group whose visual acuity losses were clearly unrelated to macular edema; the corresponding percentage was therefore based on a sample size of 120 patients. Within the nepafenac group, 2 patients developed posterior capsular opacification, I patient had clinically significant superficial punctate keratitis, and I patient had a vitreous hemorrhage that caused a 74-letter decrease in visual acuity. There were no documented confounding reasons for visual acuity loss among patients in the vehicle group.

The percentage of patients whose central subfield macular thickness values returned to baseline was also analyzed and is presented in Table 6 . Given that the coefficient of variation is $10 \%$ for the Stratus OCT 3, a patient was considered to have returned to baseline if the central subfield value was within $10 \%$ of the baseline value. A total of $78 \%$ of patients in the nepafenac group returned to baseline by the day 60 visit, in contrast with $55 \%$ of patients in the vehicle group.

The primary efficacy endpoint evaluated the number and percentage of patients who developed macular edema within 90 days postsurgery based on an increase of $30 \%$ or more in central subfield macular thickness measurements; an additional analysis was conducted using the center point macular thickness measurements. Overall, a significantly greater percentage of patients developed macular edema based on center point macular thickness in the vehicle group than in the nepafenac group (35 of 126 patients [27.8\%] versus 15 of 125 patients [12.0\%], respectively; $P=0.002$, data not presented in tables/figures). With the exception of one patient in the nepafenac group, all of the patients who met the threshold for development of macular edema based on central subfield macular thickness measurements also met the threshold for development of macular edema based on center point macular thickness measurements.

All of the patients in the study received adjunctive steroid therapy for 2 weeks postsurgery, approximately onethird of the patients were instructed, based on the opinion of the investigator, to use steroids for more than 2 weeks postsurgery. Overall, no significant differences in the key study endpoints were observed based on a comparison of steroid dosing durations (data not shown in tables/figures). Specifically, the percentages of patients in the nepafenac group who developed macular edema within 90 days postsurgery and who used steroids for more than 2 weeks or who used steroids for only 2 weeks were not significantly different $(0.0 \%$ versus $4.7 \%$, respectively; $P=0.305)$. Within the vehicle group, the corresponding percentages were also not

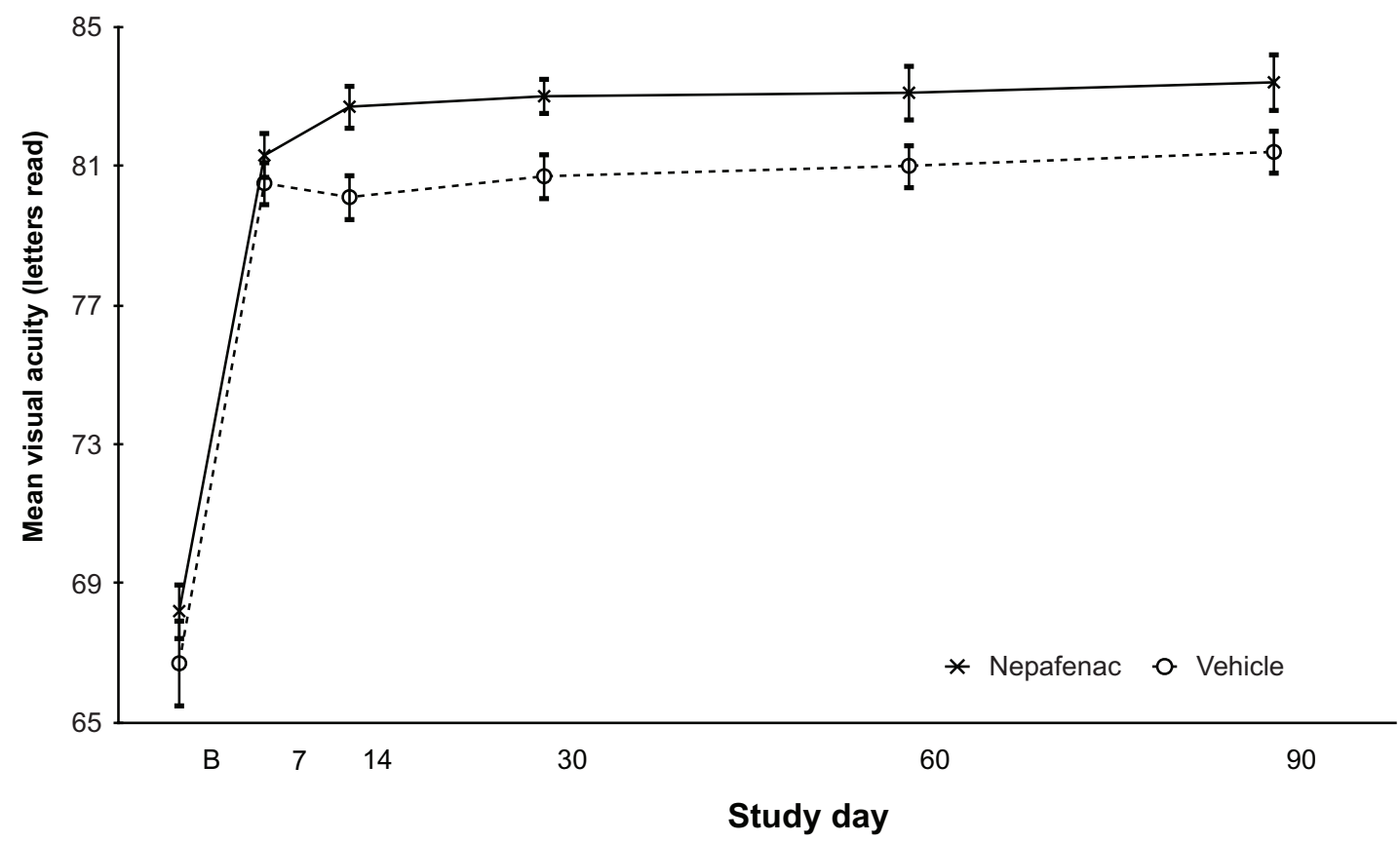

Figure 3 Mean best-corrected visual acuity (intent-to-treat data).

Notes: Data are presented as the mean number of letters read \pm standard error. The study day identified as "B" represents the presurgical baseline. No $P$-values were calculated. The sample sizes in the nepafenac group were I 25 (baseline, and days I4, 30, 60, and 90) and I 24 (day 7 ); the sample sizes in the vehicle group were I 24 (baseline), 123 (days 7 and 30), 122 (day 14), and I 25 (days 60 and 90). 
Table 4 Overall changes in best-corrected visual acuity

\begin{tabular}{|c|c|c|c|c|}
\hline & & Nepafenac & Vehicle & $P$-value \\
\hline $\begin{array}{l}\text { Absolute changes } \\
\text { (from postsurgical baseline } \\
\text { [day 7] to early exit } \\
\text { [day 90]) }\end{array}$ & $N$ & 124 & 122 & \\
\hline $\begin{array}{l}\text { Mean letters read }(\mathrm{SD}) \\
\text { (minimum, maximum) }\end{array}$ & & $\begin{array}{l}2.1(8.8) \\
(-74,23)\end{array}$ & $\begin{array}{l}0.9(6.7) \\
(-26,23)\end{array}$ & 0.226 \\
\hline $\begin{array}{l}\text { Categorical changes } \\
\text { (from presurgical baseline } \\
\text { to early exit [day 90]) }\end{array}$ & $N$ & 125 & 124 & \\
\hline Increase (n, \%) & & & & \\
\hline$\geq 15$ letters read & & 7I (56.8) & $52(41.9)$ & 0.019 \\
\hline 10-14 letters read & & $19(15.2)$ & $27(21.8)$ & \\
\hline No change (n, \%) & & & & \\
\hline 5-9 letters read & & $16(12.8)$ & $20(16.1)$ & \\
\hline \pm 4 letters read & & $17(13.6)$ & $18(14.5)$ & \\
\hline Decrease $(n, \%)$ & & & & \\
\hline 5-9 letters read & & $\mathrm{I}(0.8)$ & $5(4.0)$ & \\
\hline 10-14 letters read & & $0(0.0)$ & $0(0.0)$ & \\
\hline$\geq 15$ letters read & & $\mathrm{I}(0.8)$ & $2(1.6)$ & \\
\hline
\end{tabular}

Abbreviation: SD, standard deviation.

significantly different $(20.8 \%$ versus $11.5 \% ; P=0.140)$. Similar observations were made based on the percentages of patients in each treatment group who had best-corrected visual acuity decreases of more than five letters from the postsurgical baseline to day 90 and who used steroids for more than 2 weeks or for only 2 weeks (nepafenac, 5.0\% versus $6.0 \%$, respectively; $P=1.000$; vehicle, $10.9 \%$ versus $11.8 \%$, respectively; $P=0.870$ ). Therefore, these results

Table 5 Development of macular edema within 90 days of cataract surgery by demographic subgroup

\begin{tabular}{|c|c|c|c|c|}
\hline & \multicolumn{2}{|c|}{ Nepafenac } & \multicolumn{2}{|c|}{ Vehicle } \\
\hline & Total & n (\%) & Total & n (\%) \\
\hline \multicolumn{5}{|l|}{ Age } \\
\hline Adults & 46 & $0(0.0)$ & 54 & $8(14.8)$ \\
\hline Elderly & 79 & $4(5.1)$ & 72 & $13(18.1)$ \\
\hline \multicolumn{5}{|l|}{ Sex } \\
\hline Female & 83 & $4(4.8)$ & 75 & II (I4.7) \\
\hline Male & 42 & $0(0.0)$ & 51 & $10(19.6)$ \\
\hline \multicolumn{5}{|l|}{ Ethnicity } \\
\hline Hispanic, Latino, or Spanish & 42 & $2(4.8)$ & 45 & $7(15.6)$ \\
\hline $\begin{array}{l}\text { Not Hispanic, Latino, } \\
\text { or Spanish }\end{array}$ & 83 & $2(2.4)$ & 81 & $14(17.3)$ \\
\hline \multicolumn{5}{|l|}{ NPDR classification } \\
\hline Mild NPDR & 33 & I (3.0) & 32 & $4(12.5)$ \\
\hline Moderate NPDR & 82 & $2(2.4)$ & 83 & $14(16.9)$ \\
\hline Severe NPDR & 10 & I (I0.0) & 11 & $3(27.3)$ \\
\hline
\end{tabular}

Note: Macular edema was defined as $\geq 30 \%$ increase from the presurgical baseline in central subfield macular thickness as measured by optical coherence tomography (Stratus OCT 3).

Abbreviation: NPDR, nonproliferative diabetic retinopathy.
Table 6 Percent of patients with central subfield macular thickness values within $\pm 10 \%$ of baseline at day 60 and day 90

\begin{tabular}{llllll}
\hline & Day 60 & & & Day 90 & \\
\cline { 2 - 3 } & Total & $\mathbf{n}(\%)$ & & Total & $\mathbf{n}(\%)$ \\
\hline Nepafenac & 118 & $92(78.0)$ & & 117 & $93(79.5)$ \\
Vehicle & 115 & $64(55.7)$ & & 102 & $65(63.7)$ \\
\hline
\end{tabular}

Note: No imputations were made for missing data.

indicated that there was no added benefit to dosing steroids for more than 2 weeks postsurgery.

\section{Safety}

No patient deaths were reported during the study. Overall, 13 patients reported other serious adverse events, none of which were related to treatment. Three of the serious adverse events reported in the vehicle group (cardiac failure congestive, coronary artery occlusion, and pancreatitis) led to patient discontinuation; no other serious adverse events led to discontinuation in either treatment group. Separate from the three patients who discontinued due to serious adverse events, four other patients discontinued study participation due to nonserious adverse events. Of these nonserious events, two reported instances of punctate keratitis (one in each treatment group) were assessed as being related to the study drugs. No instances of targeted adverse events (defined as corneal erosions) were reported during the study.

Two reports of punctate keratitis and a single report of corneal epithelium defect were assessed as being related to treatment with nepafenac. A single report of punctate keratitis was assessed as being related to treatment with vehicle. No other ocular or nonocular adverse events reported in the study were assessed as being related to the study drugs. Overall, a review of adverse events revealed no safety issues based upon assessments of incidence, seriousness, relationship to the study drug, onset, outcome, duration, severity, and patient discontinuation due to adverse events.

In both treatment groups, corneal staining and intraocular pressure were each generally similar at the presurgical baseline and at the day 90 visit (or early exit). Additionally, no safety issues or trends were identified based upon changes from baseline in fundus parameters (retina/macula/choroid and optic nerve) and ocular signs (inflammatory cells, aqueous flare, corneal edema, and bulbar conjunctival injection). The study results indicate no new clinically relevant risks associated with increasing the dosing of nepafenac from 14 days to 90 days, even in the higher-risk diabetic patient population. 


\section{Discussion}

This prospective, randomized clinical study demonstrated that, based on retinal thickening and vision, treatment with nepafenac beginning presurgically and used for up to 90 days after cataract extraction was associated with a reduction in risk of macular edema and associated loss of visual acuity in diabetic patients. In this study, fewer patients in the nepafenac group developed macular edema relative to patients in the vehicle group, with significant differences seen as early as 30 days after cataract surgery, which persisted for 60 days and 90 days after surgery. The results further showed that patients in the nepafenac group, when compared with those in the vehicle group, maintained better visual acuity, with fewer decreases over the course of the study. In addition, from the postsurgical baseline to day 90 , a significantly larger percentage of patients in the nepafenac group than in the vehicle group had increases of 15 letters or more in best-corrected visual acuity.

This study was conducted in diabetic patients because it is well documented that macular changes are more likely to occur following cataract surgery in these patients, especially if they have pre-existing retinopathies, when compared with nondiabetic patients. ${ }^{5-8}$ In the present study, the observed cumulative incidence of macular edema among patients in the vehicle group was $16.7 \%$. This estimate is lower than previous studies that have generally reported incidences of $31 \%-81 \%$ in diabetic patients (with or without diabetic retinopathy) at various time points following cataract extraction. ${ }^{9-12}$ Given that the macular edema rates reported in the literature for diabetic retinopathy patients vary considerably based on the criteria used to define macular edema, the severity of retinopathy in the study population at baseline, the presence or absence of pre-existing macular edema among enrolled patients, the number of patients and time points evaluated, and the methods used to assess macular edema, it is not surprising that the rates reported in this study are different from the rates reported in some previous studies. Although the incidence of macular edema in the vehicle group was lower than predicted, the primary objective of this study was to evaluate the treatment effect, and the results for the nepafenac group were both statistically significant and clinically relevant.

The definition of macular edema used in this study is an appropriate threshold for a clinically relevant change and is well above the $10 \%$ coefficient of variation associated with repeated OCT testing. ${ }^{23,24}$ Further, because OCT provides reproducible measurements of macular thickness and volume in normal individuals and in diabetic patients with or without retinopathy, ${ }^{25-29}$ this method was considered appropriate to assess changes in macular thickness. When compared with stereoscopic biomicroscopy, which is a subjective evaluation dependent on multiple factors (eg, observer skill, patient cooperation, pupil dilation, clarity of ocular media, and characteristics of retinal swelling), OCT provides an objective and sensitive approach for measuring changes in macular thickness and volume. ${ }^{30}$

For evaluation of best-corrected visual acuity, the postsurgical evaluation on day 7 was considered the baseline assessment. The designation of day 7 as baseline in these analyses was based on several factors. In particular, by 7 days postsurgery, most patients will have attained vision improvements associated with cataract removal, but will not have had sufficient time to develop macular edema. Thus, changes in best-corrected visual acuity that occur more than 7 days after surgery are unlikely to be related to the surgical procedure.

In addition to preventing macular edema and the associated loss of visual acuity, increases in macular thickening and volume were also observed to be less severe in patients who received nepafenac than in patients who received vehicle. These results are consistent with previous clinical studies and further support the conclusion that nepafenac effectively reduced the incidence and severity of macular edema. For example, in a retrospective study based upon chart reviews of 450 patients, although visually significant pseudophakic macular edema occurred in patients treated for 4 weeks following cataract surgery either with a combination of nepafenac and prednisolone or with prednisolone alone, the percentage of patients who developed macular edema was significantly lower in the combination therapy group than in the monotherapy group $(0 \%$ and $2 \%$, respectively; $P=0.035) .{ }^{31}$ Additionally, two published case series have reported reduction in macular thickness and improvement in visual acuity following treatment with nepafenac in patients with diabetic macular edema secondary to either cataract surgery or various retinal diseases. ${ }^{32-34}$

In this study, the beneficial effect of nepafenac in preventing macular edema and associated loss of visual acuity was also supported by the decreased extent of mean macular thickening and volume observed at all postoperative study visits in the nepafenac group relative to the vehicle group. Overall, macular edema in the nepafenac group returned to baseline in a majority of patients (78\%) by postoperative day 60 . However, in the vehicle group, $63 \%$ of patients with macular edema returned to baseline by day 90 .

The central subfield measurements were based on a circular area with a $1 \mathrm{~mm}$ diameter located around the center 
point of the fovea, while the center point measurements were based on the intersection of the six radial scans obtained using the fast macular thickness protocol from OCT. In general, central subfield and center point measurements were well correlated, but in one study, central subfield macular thickness had greater reproducibility, was often better correlated with other central macular measurements, and was thought to be the preferred evaluation method. ${ }^{35}$ However, in this study, there were no important differences in the results when macular thickness was measured by center point rather than by central subfield.

No efficacy trends were observed based on patient demographics (age, sex, or ethnicity) or whether adjunctive steroid therapy was administered for 2 weeks or for more than 2 weeks following cataract surgery. While a majority of the patients had moderate nonproliferative diabetic retinopathy at baseline, the incidence of macular edema increased with increasing severity of nonproliferative diabetic retinopathy in both treatment groups. The increases were lower than expected among patients in the nepafenac group, while more substantial increases were observed among patients in the vehicle group. Although all patients were treated with steroids for 2 weeks postsurgery, approximately one-third of the patients were treated with steroids for more than 2 weeks postsurgery. However, no significant differences in the key study endpoints were observed in either treatment group based solely on steroid dosing durations.

The only treatment-related adverse events associated with nepafenac included two reports of punctate keratitis (one of which resulted in patient discontinuation) and one report of a corneal epithelium defect. These ocular events are consistent with previous clinical study experience with nepafenac. Because this was the first large study conducted in diabetic patients who had extended exposure to nepafenac, the potential for corneal problems was considered a safety concern. Corneal effects, including keratitis, are not unexpected and have been documented in previous clinical studies and in postmarketing experience with nepafenac. ${ }^{36}$ Because of the class effects for NSAIDs, corneal erosion was evaluated specifically, and exclusion criteria were added to the protocol to ensure that patients with compromised corneas were not enrolled. None of the patients in either treatment group experienced corneal erosions during the current study. Further, corneal staining assessments and intraocular pressure measurements did not change appreciably in either treatment group over the course of the study and no safety issues or trends were identified based upon changes from baseline in fundus parameters or ocular signs. Overall, the results of the study indicate that there is no increased risk associated with increasing the dosing of nepafenac from 14 days to 90 days postsurgery, even in the higher-risk diabetic population.

Although many cataract surgeons believe that it is beneficial to add NSAID therapy to the regimen for cataract patients to prevent macular edema, ${ }^{37}$ the low incidence of macular edema, along with the difficulties associated with fluorescein angiography, have made it difficult to establish a treatment effect. The recent availability of OCT has made this type of evaluation possible. In an effort to conduct a reasonably sized clinical study, only patients with diabetic retinopathy were included in the current study because these patients are known to have higher incidences of macular edema following cataract surgery. This was an appropriately enriched population because inflammatory pathways were inhibited in a similar manner in diabetics and nondiabetics. In summary, the current study supports the beneficial effects of topical ocular therapy with nepafenac and further demonstrates that topical ocular therapy with nepafenac is effective in the prevention of macular edema and associated loss of visual acuity following cataract surgery. Combined with no clinically relevant increase in risk with dosing for 90 days, the risk/benefit to the diabetic patient undergoing cataract surgery is clearly positive.

\section{Disclosures}

RS, LA, GJJ, RPL, JL, HJR, KS, and TW are paid consultants for Alcon Research Ltd (Fort Worth, TX). DS is an employee of Alcon Research, Ltd. Medical writing support, which was funded by Alcon Research Ltd, was provided by Cullen T Vogelson and Usha Sivaprasad, of Illuminated Research LLC (Fort Worth, TX). The principal author presented selected data at American Society of Cataract and Refractive Surgery annual meeting, San Diego, CA, March 25-29, 2011.

\section{References}

1. Tranos PG, Wickremasinghe SS, Stangos NT, Topouzis T, Tsinopoulos I, Pavesio CE. Macular edema. Surv Ophthalmol. 2004;49:470-490.

2. Gallemore RP. NSAIDs in treatment of retinal disorders. Rev Ophth. 2006;13:81-88.

3. Powe NR, Schein OD, Gieser SC, et al. Synthesis of the literature on visual acuity and complications following cataract extraction with intraocular lens implantation. Cataract Patient Outcome Research Team. Arch Ophthalmol. 1994;112:239-252.

4. Ursell PG, Spalton DJ, Whitcup SM, Nussenblatt RB. Cystoid macular edema after phacoemulsification: relationship to blood-aqueous barrier damage and visual acuity. J Cataract Refract Surg. 1999;25: 1492-1497.

5. Hayashi K, Igarashi C, Hirata A, Hayashi H. Changes in diabetic macular oedema after phacoemulsification surgery. Eye (Lond). 2009;23: 389-396. 
6. Degenring RF, Vey S, Kamppeter B, Budde WM, Jonas JB, Sauder G. Effect of uncomplicated phacoemulsification on the central retina in diabetic and non-diabetic subjects. Graefes Arch Clin Exp Ophthalmol. 2007;245:18-23.

7. Rossetti L, Autelitano A. Cystoid macular edema following cataract surgery. Curr Opin Ophthalmol. 2000;11:65-72.

8. Johnson MW. Etiology and treatment of macular edema. Am J Ophthalmol. 2009;147:11-21.

9. Dowler JG, Sehmi KS, Hykin PG, Hamilton AM. The natural history of macular edema after cataract surgery in diabetes. Ophthalmology. 1999;106:663-668.

10. Pollack A, Leiba H, Bukelman A, Oliver M. Cystoid macular oedema following cataract extraction in patients with diabetes. Br JOphthalmol. 1992;76:221-224.

11. Pollack A, Leiba H, Bukelman A, Abrahami S, Oliver M. The course of diabetic retinopathy following cataract surgery in eyes previously treated by laser photocoagulation. Br J Ophthalmol. 1992;76:228-231.

12. Krepler K, Biowski R, Schrey S, Jandrasits K, Wedrich A. Cataract surgery in patients with diabetic retinopathy: visual outcome, progression of diabetic retinopathy, and incidence of diabetic macular oedema. Graefes Arch Clin Exp Ophthalmol. 2002;240:735-738.

13. Miyake K, Ibaraki N. Prostaglandins and cystoid macular edema. Surv Ophthalmol. 2002;47 Suppl 1:S203-S218

14. Flach AJ. The incidence, pathogenesis and treatment of cystoid macular edema following cataract surgery. Trans Am Ophthalmol Soc. 1998;96:557-634.

15. O'Brien TP. Emerging guidelines for use of NSAID therapy to optimize cataract surgery patient care. Curr Med Res Opin. 2005;21 1131-1137.

16. Rossetti L, Chaudhuri J, Dickersin K. Medical prophylaxis and treatment of cystoid macular edema after cataract surgery. The results of a meta-analysis. Ophthalmology. 1998;105:397-405.

17. Henderson BA, Kim JY, Ament CS, Ferrufino-Ponce ZK, Grabowska A, Cremers SL. Clinical pseudophakic cystoid macular edema. Risk factors for development and duration after treatment. J Cataract Refract Surg. 2007;33:1550-1558.

18. Gaynes BI, Onyekwuluje A. Topical ophthalmic NSAIDs: a discussion with focus on nepafenac ophthalmic suspension. Clin Ophthalmol. 2008;2:355-368.

19. Ke TL, Graff G, Spellman JM, Yanni JM. Nepafenac, a unique nonsteroidal prodrug with potential utility in the treatment of trauma-induced ocular inflammation: II. In vitro bioactivation and permeation of external ocular barriers. Inflammation. 2000;24:371-384.

20. Lane SS. Nepafenac: a unique nonsteroidal prodrug. Int Ophthalmol Clin. 2006;46:13-20.

21. Kapin MA, Yanni JM, Brady MT, et al. Inflammation-mediated retinal edema in the rabbit is inhibited by topical nepafenac. Inflammation. 2003;27:281-291.

22. Wilkinson CP, Ferris FL 3rd, Klein RE, et al. Proposed international clinical diabetic retinopathy and diabetic macular edema disease severity scales. Ophthalmology. 2003;110:1677-1682.
23. Kim SJ, Belair ML, Bressler NM, et al. A method of reporting macular edema after cataract surgery using optical coherence tomography. Retina. 2008;28:870-876.

24. Kim SJ, Equi R, Bressler NM. Analysis of macular edema after cataract surgery in patients with diabetes using optical coherence tomography. Ophthalmology. 2007;114:881-889.

25. Hee MR, Puliafito CA, Duker JS, et al. Topography of diabetic macular edema with optical coherence tomography. Ophthalmology. 1998;105: 360-370.

26. Hee MR, Puliafito CA, Wong C, et al. Quantitative assessment of macular edema with optical coherence tomography. Arch Ophthalmol. 1995;113:1019-1029.

27. Goebel W, Kretzchmar-Gross T. Retinal thickness in diabetic retinopathy: a study using optical coherence tomography (OCT). Retina. 2002;22:759-767.

28. Goebel W, Franke R. Retinal thickness in diabetic retinopathy: comparison of optical coherence tomography, the retinal thickness analyzer, and fundus photography. Retina. 2006;26:49-57.

29. Campbell RJ, Coupland SG, Buhrmann RR, Kertes PJ. Optimal optical coherence tomography-based measures in the diagnosis of clinically significant macular edema: retinal volume vs foveal thickness. Arch Ophthalmol. 2007;125:619-623.

30. Bressler NM, Edwards AR, Antoszyk AN, et al. Retinal thickness on Stratus optical coherence tomography in people with diabetes and minimal or no diabetic retinopathy. Am J Ophthalmol. 2008;145: 894-901.

31. Wolf EJ, Braunstein A, Shih C, Braunstein RE. Incidence of visually significant pseudophakic macular edema after uneventful phacoemulsification in patients treated with nepafenac. J Cataract Refract Surg 2007;33:1546-1549.

32. Hariprasad SM, Akduman L, Clever JA, Ober M, Recchia FM, Mieler WF. Treatment of cystoid macular edema with the newgeneration NSAID nepafenac 0.1\%. Clin Ophthalmol. 2009;3: 147-154.

33. Hariprasad SM, Callanan D, Gainey S, He YG, Warren K. Cystoid and diabetic macular edema treated with nepafenac $0.1 \%$.J Ocul Pharmacol Ther. 2007;23:585-590.

34. Callanan D, Williams P. Topical nepafenac in the treatment of diabetic macular edema. Clin Ophthalmol. 2008;2:689-692.

35. Krzystolik MG, Strauber SF, Aiello LP, et al; Diabetic Retinopathy Clinical Research Network. Reproducibility of macular thickness and volume using Zeiss optical coherence tomography in patients with diabetic macular edema. Ophthalmology. 2007;114:1520-1525.

36. Nevanac ${ }^{\circledR}$ (full prescribing information). Fort Worth, TX: Alcon Laboratories Inc; 2008.

37. Heier JS, Topping TM, Baumann W, Dirks MS, Chern S. Ketorolac versus prednisolone versus combination therapy in the treatment of acute pseudophakic cystoid macular edema. Ophthalmology. 2000;107:2034-2038.
Clinical Ophthalmology

\section{Publish your work in this journal}

Clinical Ophthalmology is an international, peer-reviewed journal covering all subspecialties within ophthalmology. Key topics include: Optometry; Visual science; Pharmacology and drug therapy in eye diseases; Basic Sciences; Primary and Secondary eye care; Patient Safety and Quality of Care Improvements. This journal is indexed on Submit your manuscript here: http://www.dovepress.com/clinical-ophthalmology-journa

\section{Dovepress}

PubMed Central and CAS, and is the official journal of The Society of Clinical Ophthalmology (SCO). The manuscript management system is completely online and includes a very quick and fair peer-review system, which is all easy to use. Visit http://www.dovepress.com/ testimonials.php to read real quotes from published authors. 